\title{
Coherent Backscattering in Fock Space: A Signature of Quantum Many-Body Interference in Interacting Bosonic Systems
}

\author{
Thomas Engl, ${ }^{1}$ Julien Dujardin, ${ }^{2}$ Arturo Argüelles, ${ }^{2}$ Peter Schlagheck, ${ }^{2}$ Klaus Richter, ${ }^{1}$ and Juan Diego Urbina ${ }^{1}$ \\ ${ }^{1}$ Institut für Theoretische Physik, Universität Regensburg, D-93040 Regensburg, Germany \\ ${ }^{2}$ Département de Physique, University of Liege, 4000 Liège, Belgium
}

(Received 12 June 2013; published 9 April 2014)

\begin{abstract}
We predict a generic signature of quantum interference in many-body bosonic systems resulting in a coherent enhancement of the average return probability in Fock space. This enhancement is robust with respect to variations of external parameters even though it represents a dynamical manifestation of the delicate superposition principle in Fock space. It is a genuine quantum many-body effect that lies beyond the reach of any mean-field approach. Using a semiclassical approach based on interfering paths in Fock space, we calculate the magnitude of the backscattering peak and its dependence on gauge fields that break time-reversal invariance. We confirm our predictions by comparing them to exact quantum evolution probabilities in Bose-Hubbard models, and discuss their relevance in the context of many-body thermalization.
\end{abstract}

DOI: 10.1103/PhysRevLett.112.140403

PACS numbers: 03.65.Yz, 05.45.Mt, 67.85.-d, 72.15.Rn

The existence of a superposition principle for quantum states is a cornerstone in our picture of the physical world, with observable implications in the form of coherent phenomena that have been experimentally demonstrated with impressive precision during the last century [1-3]. Within the context of linear wave equations, quantum superposition effects represent particular cases of the general phenomenon of wave coherence. For quantum systems described by the single-particle Schrödinger equation, this analogy between quantum and classical waves was exploited to demonstrate coherent quantum effects, such as Anderson localization in disordered metals [4] or coherent backscattering (CBS) [5], by using classical (in particular electromagnetic) wave analogues [6-8].

In the quantum description of many-body systems, such an analogy between quantum dynamics and classical wave phenomena does not hold. Within a first-quantized approach, the quantum mechanical description of a system of $N$ interacting particles in $D$ dimensions requires us to extend the space in which the Schrödinger field $\psi\left(\vec{r}_{1}, \ldots, \vec{r}_{N}, t\right)$ is defined to $N D$ dimensions. We can still identify the quantum superposition principle with the linearity of the many-body Schrödinger equation, but the latter does no longer describes a classical wave in real $D$-dimensional space: many-body quantum interference is a high-dimensional phenomenon.

This observation remains true even if we adopt a realspace description in terms of the quantum field $\hat{\psi}(\vec{r}, t)$. Indeed, $\hat{\psi}(\vec{r}, t)$ is an operator instead of a complex amplitude and does not represent a quantum state. Nevertheless, quantum fields are a suitable starting point to implement approximations to the full many-body problem in terms of classical wave equations for single particles, which effectively amounts to the substitution $\hat{\psi}(\vec{r}, t) \rightarrow \psi(\vec{r}, t)$ at the level of the Heisenberg equations of motion for $\hat{\psi}(\vec{r}, t)$. This approach leads to the mean-field Gross-Pitaevskii equation (GPE) [9] and also to the truncated Wigner method [10-13] with its quantum (Wigner-Moyal) corrections [14]. Since the GPE is a classical field equation, its nonlinearity does not pose a conflict with the linearity of quantum evolution. For the same reasons, however, the $\psi(\vec{r}, t)$ field cannot represent a quantum state and its physical meaning requires further interpretation as a condensate fraction or order parameter. In particular, interference effects resulting from the (weakly nonlinear) GPE are not a consequence of many-body interference; they are classical wave effects proper of a classical field equation and are generically suppressed already for small interactions [15-18].

In this Letter we report a semiclassical description of the quantum mechanism responsible for many-body interference phenomena in interacting bosonic systems, which is schematically illustrated in Fig. 1. Our approach is based on coherent sums over multiple solutions of the GPE in occupation number space. It predicts quantum coherence effects that are in quantitative agreement with numerical simulations of Bose-Hubbard models describing cold atoms in optical lattices.

Since many-body interference is most visible in genuine many-body observables (i.e., which cannot be written as expectation values of single-particle operators), we will study, as a representative example, the microscopic evolution probability from one many-body state to another one. Following standard techniques [19], we introduce a discrete and orthogonal but otherwise arbitrary set of $L$ single-particle states ("orbitals") $\chi_{1}, \ldots, \chi_{L}$. The associated set of commuting bosonic occupation number operators $\hat{n}_{\alpha}$ has common (Fock) eigenstates $|\mathbf{n}\rangle=\left|n_{1}, \ldots, n_{L}\right\rangle$ and integer eigenvalues $n_{\alpha}$ denoting the number of particles in each orbital $\chi_{\alpha}$. The transition probability between Fock states at time $t$ reads then 


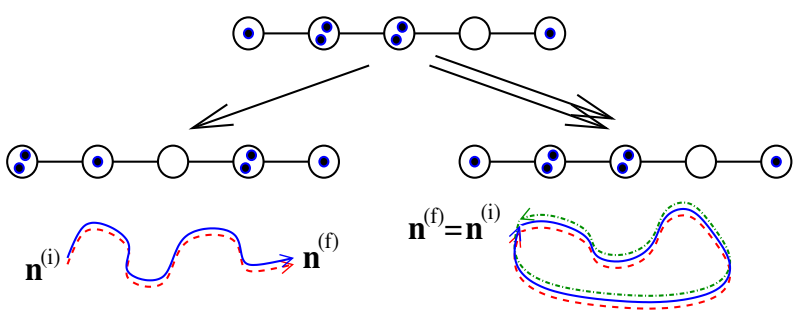

FIG. 1 (color online). Illustration of coherent backscattering in Fock space. A many-body system, represented here by a BoseHubbard chain with $L=5$ sites and $N=6$ particles, is prepared in a well-defined initial Fock state $n^{(\mathrm{i})}=\left(n_{1}^{(\mathrm{i})}, \ldots, n_{L}^{(\mathrm{i})}\right)$ (upper part). After a given evolution time $t$, the final populations are found to be $n^{(\mathrm{f})}$ (lower parts). A solution $\gamma$ of the Gross-Pitaevskii equation joining $n^{(\mathrm{i})}$ with $n^{(\mathrm{f})}$ contributes with an amplitude $K_{\gamma} \simeq A_{\gamma} e^{i R_{\gamma}}$ to this process, where $R_{\gamma}$ is a classical action. Under averaging, only pairs of identical Fock space trajectories yield a systematically nonvanishing contribution to the probability $P=\left|\sum_{\gamma} K_{\gamma}\right|^{2}$ when $n^{(\mathrm{f})} \neq n^{(\mathrm{i})}$ (left column). For $n^{(\mathrm{f})}=n^{(\mathrm{i})}$ (right column), however, constructive interference additionally arises if trajectories are paired with their time-reversed counterparts (dot-dashed green line). This gives rise to a coherent enhancement of the probability to detect the system in the initial Fock state after the evolution, as compared to other states with comparable distributions of the population.

$$
P\left(\mathbf{n}^{(\mathrm{f})}, \mathbf{n}^{(\mathrm{i})}, t\right)=\left|\left\langle\mathbf{n}^{(\mathrm{f})}|\hat{U}(t)| \mathbf{n}^{(\mathrm{i})}\right\rangle\right|^{2},
$$

with $\hat{U}(t) \equiv \exp (-i \hat{H} t / \hbar)$ and the general many-body Hamiltonian exhibiting two-body interaction

$$
\hat{H}=\sum_{\alpha \beta} h_{\alpha \beta} \hat{a}_{\alpha}^{\dagger} \hat{a}_{\beta}+\frac{1}{2} \sum_{\alpha \beta \eta \sigma} V_{\alpha \beta \eta \sigma} \hat{a}_{\alpha}^{\dagger} \hat{a}_{\beta} \hat{a}_{\eta}^{\dagger} \hat{a}_{\sigma}
$$

which is expressed in terms of the ladder operators $\hat{a}_{\alpha}\left(\hat{a}_{\alpha}^{\dagger}\right)$ that annihilate (create) a particle in the orbital $\chi_{\alpha}$ (with $\hat{n}_{\alpha}=\hat{a}_{\alpha}^{\dagger} \hat{a}_{\alpha}$ ). We shall later on refer to the more specific case of the Bose-Hubbard (BH) model

$$
\begin{aligned}
\hat{H}_{\mathrm{BH}}= & \sum_{\alpha} \epsilon_{\alpha} \hat{n}_{\alpha}-J \sum_{\alpha}\left(e^{i \phi} \hat{a}_{\alpha}^{\dagger} \hat{a}_{\alpha+1}+e^{-i \phi} \hat{a}_{\alpha+1}^{\dagger} \hat{a}_{\alpha}\right) \\
& +\frac{U}{2} \sum_{\alpha} \hat{n}_{\alpha}\left(\hat{n}_{\alpha}-1\right),
\end{aligned}
$$

which describes, e.g., cold atoms in optical lattices.

Our calculation (see the Supplemental Material [20] for details) is based on an asymptotic expansion of the manybody propagator,

$$
K^{\mathrm{sc}}\left(\mathbf{n}^{(\mathrm{f})}, \mathbf{n}^{(\mathrm{i})}, t\right) \simeq\left\langle\mathbf{n}^{(\mathrm{f})}|\hat{U}(t)| \mathbf{n}^{(\mathrm{i})}\right\rangle,
$$

which is formally valid for $n_{\alpha}^{(\mathrm{i}, \mathrm{f})} \gg 1$. To this end, we have to consider all solutions (indexed by $\gamma$ )

$$
\boldsymbol{\psi}^{(\gamma)}(s) \equiv \boldsymbol{\psi}^{(\gamma)}\left(s ; \mathbf{n}^{(\mathrm{f})}, \mathbf{n}^{(\mathrm{i})}, t\right) \equiv\left[\psi_{1}^{(\gamma)}(s), \ldots, \psi_{L}^{(\gamma)}(s)\right]
$$

of the mean-field GPE

$$
i \hbar \frac{\partial}{\partial s} \psi_{\alpha}=\sum_{\beta} h_{\alpha \beta} \psi_{\beta}+\sum_{\beta \eta \sigma} V_{\alpha \beta \eta \sigma} \psi_{\beta} \psi_{\eta}^{*} \psi_{\sigma}
$$

that instead of initial conditions satisfy the bilateral boundary (or shooting) conditions $\left|\psi_{\alpha}(0)\right|^{2}=n_{\alpha}^{(\mathrm{i})}+1 / 2$ and $\left|\psi_{\alpha}(t)\right|^{2}=n_{\alpha}^{(\mathrm{f})}+1 / 2$ and have $\arg \psi_{\alpha=1}(0)=0$ (see the Supplemental Material [20]). In terms of those solutions $\boldsymbol{\psi}^{(\gamma)}(s)$, the semiclassical propagator is then expressed as

$$
K^{\mathrm{sc}}\left(\mathbf{n}^{(\mathrm{f})}, \mathbf{n}^{(\mathrm{i})}, t\right)=\sum_{\gamma} A^{(\gamma)} \exp \left[i R^{(\gamma)}+i \pi \Phi^{(\gamma)} / 4\right],
$$

where, for each solution, the semiclassical amplitude

$$
A^{(\gamma)}\left(\mathbf{n}^{(\mathrm{f})}, \mathbf{n}^{(\mathrm{i})}, t\right)=\sqrt{\left|\operatorname{det}^{\prime} \frac{1}{2 \pi} \frac{\partial^{2} R^{(\gamma)}\left(\mathbf{n}^{(\mathrm{f})}, \mathbf{n}^{(\mathrm{i})}, t\right)}{\partial \mathbf{n}^{(\mathrm{f})} \partial \mathbf{n}^{(\mathrm{i})}}\right|}
$$

is given by the (dimensionless) classical action

$$
R^{(\gamma)}=\int_{0}^{t}\left(\sum_{\alpha} \theta_{\alpha}^{(\gamma)}(s) \dot{I}_{\alpha}^{(\gamma)}(s)-H\left[\boldsymbol{\psi}^{(\gamma)}(s)\right] / \hbar\right) d s
$$

with $\psi_{\alpha}^{(\gamma)}(s) \equiv \sqrt{I_{\alpha}^{(\gamma)}(s)} \exp \left[i \theta_{\alpha}^{(\gamma)}(s)\right]$ and

$$
H(\boldsymbol{\Psi})=\sum_{\alpha \beta} h_{\alpha \beta} \psi_{\alpha}^{*} \psi_{\beta}+\frac{1}{2} \sum_{\alpha \beta \eta \sigma} V_{\alpha \beta \eta \sigma} \psi_{\alpha}^{*} \psi_{\eta}^{*} \psi_{\beta} \psi_{\sigma}
$$

the classical (mean-field) Hamiltonian. The index $\Phi^{(\gamma)}$ counts the number of conjugate points along the trajectory $\gamma$. As indicated by det', the derivatives in Eq. (8) are to be taken with respect to $n_{2}^{(\mathrm{i} / \mathrm{f})}, \ldots, n_{L}^{(\mathrm{i} / \mathrm{f})}$ with $n_{1}^{(\mathrm{i} / \mathrm{f})}$ being fixed by the total number of particles (see the Supplemental Material [20]).

The heuristic use of $H(\boldsymbol{\psi})$ as the classical limit in bosonic systems has a long history [21] and lies behind most studies of the quantum-classical correspondence in Bose-Hubbard models [12-14,22]. However, a rigorous approach in which a semiclassical propagator is constructed by a stationary phase analysis of the exact path-integral representation of $\hat{U}(t)$ in the spirit of the van VleckGutzwiller approach for first-quantized systems [23] was missing in previous studies. Importantly, our propagator $K^{\text {sc }}$ is valid also if the classical limit is nonintegrable, thus going beyond the successful WKB method of Refs. [24,25] for $L=2$ and the Einstein-Brillouin-Keller approach of Ref. [22] for $L=3$. Contrary to previous classical and quasiclassical approaches (including the standard implementations of the truncated Wigner method [10-13]), the classical information appears in Eq. (7) in terms of a boundary value problem generally exhibiting many solutions, which make interference explicit, instead of an initial value problem with a unique solution.

Substituting Eqs. (4) and (7) into Eq. (1) yields

$$
P\left(\mathbf{n}^{(\mathrm{f})}, \mathbf{n}^{(\mathrm{i})}, t\right)=\sum_{\gamma \gamma^{\prime}} A^{(\gamma)} A^{\left(\gamma^{\prime}\right)} \mathrm{e}^{i\left(R^{(\gamma)}-R^{\left(\gamma^{\prime}\right)}\right)} .
$$


From the typical scaling $R^{(\gamma)}-R^{\left(\gamma^{\prime}\right)} \propto N$ of the action differences, the contributions to the double sum in Eq. (11) contain an exponentially increasing number of highly oscillatory terms that tend to cancel each other. Averaging, e.g., over a disorder potential that is contained in the matrix elements $h_{\alpha \beta}$, then suppresses quasirandom contributions from pairs of longer, unrelated paths $\gamma \neq \gamma^{\prime}$ and selects contributions from those pairs of classical solutions that generically exhibit action quasidegeneracies: $R^{(\gamma)}-R^{\left(\gamma^{\prime}\right)} \sim 0$. The first nonvanishing contribution to the average transition probability (denoted by a bar, as is any other averaged expression) is then given by the incoherent $\left(\gamma=\gamma^{\prime}\right)$ part of the double sum,

$$
\begin{aligned}
\bar{P}^{\mathrm{cl}}\left(\mathbf{n}^{(\mathrm{f})}, \mathbf{n}^{(\mathrm{i})}, t\right)= & \sum_{\gamma} \overline{\left|A^{(\gamma)}\right|^{2}} \\
= & \int_{0}^{2 \pi} \frac{d \theta_{2}}{2 \pi} \cdots \int_{0}^{2 \pi} \frac{d \theta_{L}}{2 \pi} \\
& \times \overline{\prod_{\alpha=2}^{L} \delta\left[n_{\alpha}^{(\mathrm{f})}+\frac{1}{2}-\left|\psi_{\alpha}\left(\mathbf{n}^{(\mathrm{i})}, \boldsymbol{\theta}, t\right)\right|^{2}\right]},
\end{aligned}
$$

where $\boldsymbol{\psi}\left(\mathbf{n}^{(\mathrm{i})}, \boldsymbol{\theta}, t\right)$ is the unique solution of the GPE (6) with initial conditions satisfying $\left|\psi_{\alpha}^{(\mathrm{i})}\right|^{2}=n_{\alpha}^{(\mathrm{i})}+1 / 2$ and $\arg \psi_{\alpha}^{(\mathrm{i})}=\theta_{\alpha}$ with $\theta_{\alpha=1}=0$ [26]. Equation (12) is the averaged transition probability obtained using the classical truncated Wigner method [27].

Having identified $\bar{P}^{\mathrm{cl}}$ as the classical probability, any other robust contribution to $\bar{P}$ is necessarily a signature of many-body quantum interference. As shown schematically in Fig. 1, having exact and generic action degeneracies for $\gamma \neq \gamma^{\prime}$ requires the presence of time-reversal invariance (TRI), which means that for each solution $\boldsymbol{\psi}^{(\gamma)}(s)$ of the GPE one can find suitable phases $\omega_{\alpha}$ such that its timereversal partner $\boldsymbol{\psi}^{(\mathcal{T} \gamma)}(s)$ with

$$
\psi_{\alpha}^{(\mathcal{T} \gamma)}\left(s ; \mathbf{n}^{(\mathrm{f})}, \mathbf{n}^{(\mathrm{i})}, t\right) \equiv \mathrm{e}^{\mathrm{i} \omega_{\alpha}}\left[\psi_{\alpha}^{(\gamma)}\left(t-s ; \mathbf{n}^{(\mathrm{i})}, \mathbf{n}^{(\mathrm{f})}, t\right)\right]^{*}
$$

is also a solution of the GPE but with the initial and final conditions interchanged. In that case, it follows from Eq. (9) that $\gamma$ and $\mathcal{T} \gamma$ have the same classical actions and semiclassical amplitudes. Obviously, as the trajectories $\gamma, \gamma^{\prime}$ in the double sum (11) refer to a specific $\mathbf{n}^{(\mathrm{i})}$ and a specific $\mathbf{n}^{(\mathrm{f})}$, a pairing $\gamma^{\prime}=\mathcal{T} \gamma$ is only possible if $\mathbf{n}^{(\mathrm{f})}=\mathbf{n}^{(\mathrm{i})}$ [28]. Finally, to allow for long-path contributions such that generically $\gamma \neq \mathcal{T} \gamma$ the evolution time has to be considerably larger than the hopping time scale $\tau=\hbar / J$, which corresponds to the inverse Rabi frequency between adjacent sites (see the Supplemental Material [20]). For $t \lesssim \tau$, trajectories describing a pure phase evolution of the complex classical on-site amplitudes without altering the individual site populations dominantly contribute to $K^{\mathrm{sc}}(\mathbf{n}, \mathbf{n}, t)$. Those trajectories are, in the population space, obviously identical with their time-reversed counterpart, i.e., self-retracting, and hence no partner $\gamma^{\prime}$ with $\gamma^{\prime}=\mathcal{T} \gamma \neq \gamma$ exists.

We therefore obtain

$$
\bar{P}\left(\mathbf{n}^{(\mathrm{f})}, \mathbf{n}^{(\mathrm{i})}, t\right) \simeq \begin{cases}\bar{P}^{\mathrm{cl}}\left(\mathbf{n}^{(\mathrm{f})}, \mathbf{n}^{(\mathrm{i})}, t\right) & \text { if } t \lesssim \tau \\ (1+\delta) \bar{P}^{\mathrm{cl}}\left(\mathbf{n}^{(\mathrm{f})}, \mathbf{n}^{(\mathrm{i})}, t\right) & \text { if } t \gg \tau\end{cases}
$$

with $\delta=\delta_{\mathbf{n}^{(\mathrm{f})}, \mathbf{n}^{(\mathrm{i})}}$ in the presence of TRI and 0 otherwise. Equation (14) reflects CBS in Fock space, i.e., a coherent enhancement of the averaged quantum probability of return in Fock space over the classical value due to quantum many-body interference. Resulting from phase cancellations among oscillatory functions, this enhancement is nonperturbative in the effective Planck constant $\hbar_{\text {eff }} \sim N^{-1}$. It can be regarded as a many-body generalization of the single-particle weak localization effect in quantum transport [5], which is also of subleading order compared to the classical contribution.

To confirm our result, Eq. (14), we performed extensive numerical calculations for the BH model defined in Eq. (3) for chain and ring topologies. We defined our ensemble average through independent variations of the on-site energies $\epsilon_{\alpha}$, which are randomly selected from the interval $0<\epsilon_{\alpha}<W$. Taking advantage of the literature concerned with classical equilibration and chaos for this kind of Hamiltonian [29-31], we fixed the numerical values of the free parameters $U / J$ and $W / J$ such that the classical phase space has a dominant chaotic component. The quantum transition probability $\bar{P}\left(\mathbf{n}^{(\mathrm{f})}, \mathbf{n}^{(\mathrm{i})}, t\right)$, which corresponds to the expectation value of the many-body projector onto the Fock state $\mathbf{n}^{(\mathrm{f})}$, is then computed with a Runge-Kutta solver, using the exact quantum propagation of the initial state in full Fock space, followed by the disorder average over the on-site energies. The classical probability $\bar{P}^{\mathrm{cl}}\left(\mathbf{n}^{(\mathrm{f})}, \mathbf{n}^{(\mathrm{i})}, t\right)$, on the other hand, is directly computed from Eq. (12) where, for a given random choice of the onsite energies, $\boldsymbol{\psi}\left(\mathbf{n}^{(\mathrm{i})}, \boldsymbol{\theta}, t\right)$ is determined by the numerical solution of the $L$-dimensional GPE.

In Fig. 2 we show the time dependence of $\bar{P}$ and $\bar{P}^{\mathrm{cl}}$ as a function of $\mathbf{n}^{(\mathrm{f})}$ for the BH model (3) with $L=5, N=14$, and a chain topology (i.e., the site 1 is not connected to the site $L$ by a single hopping matrix element) starting from a generically chosen initial state $\mathbf{n}^{(i)}=(3,2,3,2,4)$. After the transient initial regime in which quantum and classical results resemble each other, the quantum transition probabilities clearly display, for $t \gtrsim 5 \tau$, a stationary CBS peak at the initial state $\mathbf{n}^{(\mathrm{i})}$ on top of a roughly constant background, in quantitative agreement with Eq. (14). This peak is not reproduced by the classical probabilities ruling out short-time effects or self-trapping due to rare realizations of the random on-site energies as alternative origins of the enhancement.

Figure 3 shows the CBS peak at $t=10 \tau$ for a BH ring (see inset) with $L=6$ sites (in which a hopping matrix element connects the site 1 to the site $L$ ) in the presence of nonvanishing hopping phases $\phi$ [see Eq. (3)], which break TRI. We clearly see the suppression of the CBS peak in the absence of TRI at $\phi=\pi / 8$ and $\pi / 4$. For $\phi=\pi / 2$, on the 


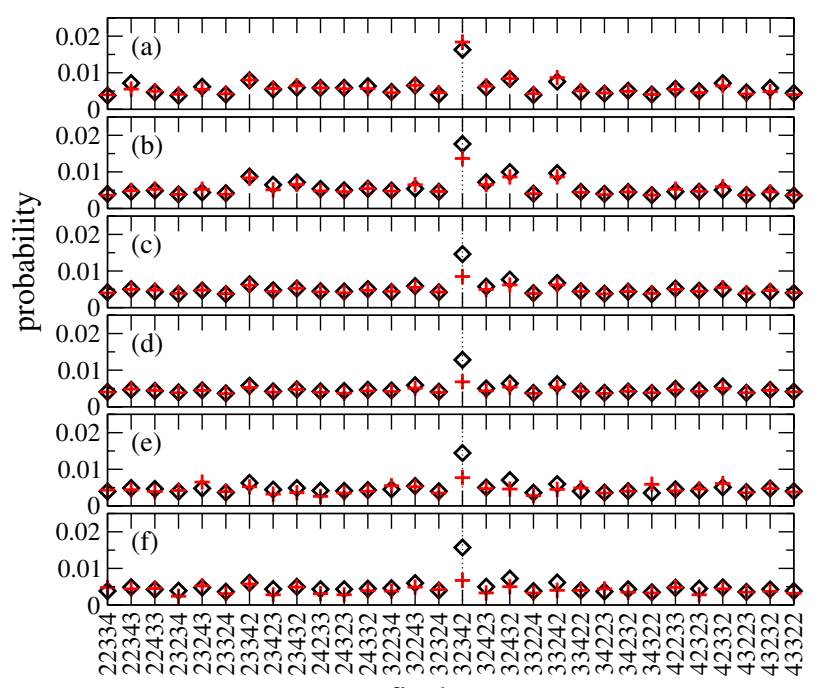

final state

FIG. 2 (color online). Average quantum (black diamonds) and classical (red crosses) evolution probabilities in Fock space for a Bose-Hubbard chain $(L=5, N=14)$, at different evolution times $t / \tau=1.5(\mathrm{a}), 2.5(\mathrm{~b}), 5(\mathrm{c}), 10(\mathrm{~d}), 20(\mathrm{e}), 50(\mathrm{f})$ with $\tau \equiv \hbar / J$. The average was performed over an ensemble of $10^{3}$ realizations of on-site energies $\epsilon_{\alpha} \in[0,10 J]$, with interaction strength $U=4 J$. The probabilities are displayed for the set of Fock states $\mathbf{n}^{(\mathrm{f})}$ having the same total interaction energy as $\mathbf{n}^{(\mathrm{i})}=(3,2,3,2,4)$ (marked by the vertical dashed line). While quantum-classical correspondence holds for $t \sim \tau$, quantum interference sets in and stabilizes for $t \gtrsim 4 \tau$ (see the Supplemental Material [20]) yielding, in accordance with Eq. (14), a systematic enhancement of the quantum backscattering probability to $\mathbf{n}^{(\mathrm{f})}=\mathbf{n}^{(\mathrm{i})}$ by about a factor 2 as compared to other final states $\mathbf{n}^{(\mathrm{f})} \neq \mathbf{n}^{(\mathrm{i})}$ and to its classical prediction.

other hand, TRI is again established using $\omega_{\alpha}=\alpha \pi$ in Eq. (13) and the CBS peak reappears.

Experimentally, CBS in many-body space could possibly be observed with ultracold bosonic atoms. The specific ring geometry of Fig. 3 could be realized in hexagonal (graphene) optical lattices [32]. A tightly focused red-detuned laser beam could be used in order to isolate an individual hexagon from the lattice. Displacing the focus of the laser beam with respect to the geometric center of this hexagon would allow one to load this ring in a nonuniform manner, i.e., such that the atomic populations differ from site to site. While the ring is initially to be loaded in the deep Mott insulator regime, in which intersite hopping along the ring is negligibly small, a sudden increase of the hopping strength at time $t=0$ will make the atoms propagate along the ring. At a given final propagation time, the system would have to be quenched back to the Mott regime and the atomic populations on the individual sites would have to be measured using, e.g., high-resolution imaging techniques [33]. Optical disorder [34] can be used to randomly vary the on-site energies in a controlled manner, and an artificial gauge field [35] could be induced in order to break TRI. The lower panel of Fig. 3 displays the numerically computed Fock state probabilities on such a ring for the

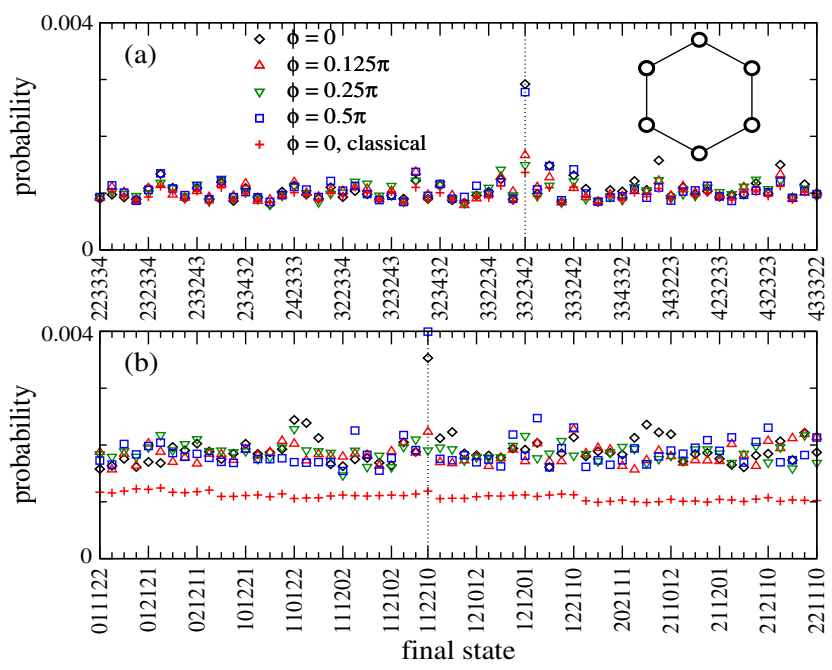

FIG. 3 (color online). Evolution probability $\bar{P}\left(\mathbf{n}^{(\mathrm{f})}, \mathbf{n}^{(\mathrm{i})}, t\right)$ in Fock space averaged over random on-site energies $\epsilon_{\alpha} \in[0, W]$ for a Bose-Hubbard ring of $L=6$ sites (see inset). We show results for evolution times $t=10 \tau$, hopping phases $\phi=0, \pi / 8, \pi / 4$, $\pi / 2$ (black diamonds, red upper triangles, green lower triangles, blue squares), and initial states $\mathbf{n}^{(\mathrm{i})}$ indicated by a vertical line. In (a) we have $N=17$ particles, with interaction strength $U=4 \mathrm{~J}$ and $W=10 J$. The lower (more "quantum") panel (b) has $N=7$, $U=J$, and $W=2 J$. In both cases, the breaking of time-reversal invariance for $\phi=\pi / 8, \pi / 4$ destroys the coherent enhancement of the backscattering probability to the initial state. In the semiclassical regime (a) the evolution probabilities globally agree with the classical prediction for $\phi=0$ (red crosses), while they significantly exceed the latter in the quantum regime (b).

initial state $\mathbf{n}^{(\mathrm{i})}=(1,1,2,2,1,0)$ [36]. It clearly displays the CBS enhancement despite the fact that this initial state is far from semiclassical $(N / L \simeq 1)$.

Our results represent a further step in the active field of thermalization in closed many-body systems [29,37-40]. Indeed, Eq. (14) shows that in equilibrium, even in the semiclassical limit and when the classical system displays full ergodicity, many-body quantum interference generically inhibits quantum ergodicity in equilibrium, i.e.,

$$
\bar{P}\left(\mathbf{n}^{(\mathrm{f})}, \mathbf{n}^{(\mathrm{i})}, t \gg t_{\mathrm{eq}}\right) \neq 1 / \mathcal{N}_{\mathrm{acc}},
$$

where $\mathcal{N}_{\text {acc }} \equiv \mathcal{N}_{\text {acc }}\left(\mathbf{n}^{(\mathrm{i})}\right)$ is the number of final Fock states that are energetically accessible to $\mathbf{n}^{(\mathbf{i})}$. This, however, is not in conflict with signatures of many-body thermalization at the level of single-particle observables, like the equilibration towards uniform occupation numbers [40], as can be easily seen by calculating the averaged values $\bar{n}_{\alpha}(t)$, which gives the expected uniform behavior

$$
\bar{n}_{\alpha}\left(t, \mathbf{n}^{(\mathrm{i})}\right)=(N / L)+\mathcal{O}\left(1 / \mathcal{N}_{\text {acc }}\right)
$$

in the regime of classical ergodicity. Clearly, Eq. (14) has a tiny effect on such single-particle observables and it is expected that a truncated Wigner approach reproduces them very well. Genuine many-body observables, on the contrary, are very sensitive to many-body interference. 
A timely example is the inverse participation ratio $\mathcal{Q}=\sum_{\mathbf{n}} \sum_{i} \overline{\left|\left\langle E_{i} \mid \mathbf{n}\right\rangle\right|^{4}}=\sum_{\mathbf{n}} \bar{P}(\mathbf{n}, \mathbf{n}, t)$ for large $t$, which is a measure of many-body localization quantifying the uniformity of Fock states in the eigenbasis $\left|E_{i}\right\rangle$ of $\hat{H}$. For TRI, a classical calculation significantly underestimates this degree of many-body localization: $\mathcal{Q}=2 \mathcal{Q}^{\text {cl }}$.

To summarize, we presented a semiclassical approach in the van Vleck-Gutzwiller spirit, using sums over interfering paths that solve a classical mean field equation, which successfully captures genuine quantum interference in interacting bosonic systems beyond the transient regime of quantumclassical correspondence. We used this approach to predict a clear-cut quantum (and genuinely many-body) effect, namely the coherent enhancement of the return probability in Fock space. Our predictions are fully confirmed by extensive simulations of Bose-Hubbard models with different topologies, even in the deep quantum regime where experimental observation using ultracold atoms is possible.

We thank A. Altland, T. Guhr, B. Gutkin, F. Haake, M. Oberthaler, W. Strunz, and T. Wellens for valuable discussions. This work was financially supported by the DFG Research Unit FOR760 as well as by a ULg research and mobility grant for T. E.

[1] A. Zeilinger, Rev. Mod. Phys. 71, S288 (1999).

[2] M. Arndt, O. Nairz, J. Vos-Andreae, C. Keller, G. van der Zouw, and A. Zeilinger, Nature (London) 401, 680 (1999).

[3] J. Deiglmayr, M. Reetz-Lamour, T. Amthor, S. Westermann, A. de Oliveira, and M. Weidemüller, Opt. Commun. 264, 293 (2006).

[4] See e.g., E. Abrahams, 50 Years of Anderson Localization (World Scientific, Singapore, 2010); reprinted in P. Wölfle and D. Vollhardt, Int. J. Mod. Phys. B 24, 1526 (2010).

[5] E. Akkermans and G. Montambaux, Mesoscopic Physics of Electrons and Photons (Cambridge University Press, Cambridge, England, 2011).

[6] E. Akkermans, P. E. Wolf, and R. Maynard, Phys. Rev. Lett. 56, 1471 (1986).

[7] F. Scheffold, R. Lenke, R. Tweer, and G. Maret, Nature (London) 398, 206 (1999).

[8] T. Schwartz, G. Bartal, S. Fishman, and M. Segev, Nature (London) 446, 52 (2007).

[9] A. L. Fetter and J. D. Walecka, Quantum Theory of ManyParticle Systems (Dover Publications, New York, 2003).

[10] C. Gardiner and P. Zoller, Quantum Noise (Springer, Berlin, 2004), 3rd ed.

[11] A. Sinatra, C. Lobo, and Y. Castin, Phys. Rev. Lett. 87, 210404 (2001).

[12] D. Walls and G. J. Milburn, Quantum Optics (Springer, New York, 2010).

[13] A. Polkovnikov, Ann. Phys. (N.Y.) 325, 1790 (2010).

[14] F. Trimborn, D. Witthaut, and H. J. Korsch, Phys. Rev. A 79, 013608 (2009).

[15] S. Flach, D. O. Krimer, and C. Skokos, Phys. Rev. Lett. 102, 024101 (2009); 102209903(E) (2009).

[16] T. Wellens and B. Gremaud, Phys. Rev. Lett. 100, 033902 (2008).
[17] M. Hartung, T. Wellens, C. A. Müller, K. Richter, and P. Schlagheck, Phys. Rev. Lett. 101, 020603 (2008).

[18] T. Hartmann, J. Michl, C. Petitjean, T. Wellens, J.-D. Urbina, K. Richter, and P. Schlagheck, Ann. Phys. (Amsterdam) 327, 1998 (2012).

[19] J. W. Negele and H. Orland, Quantum Many-Particle Systems (Addison-Wesley, Redwood City, CA, 1988).

[20] See Supplemental Material at http://link.aps.org/ supplemental/10.1103/PhysRevLett.112.140403 for the derivation of the semiclassical many-body propagator in Fock space and a discussion of the time scales involved.

[21] W. Heisenberg, Z. Phys. 33, 879 (1925).

[22] S. Mossmann and C. Jung, Phys. Rev. A 74, 033601 (2006).

[23] see M. C. Gutzwiller, Chaos in Classical and Quantum Mechanics (Springer, New York, 1990) and references therein.

[24] L. Simon and W. T. Strunz, Phys. Rev. A 86, 053625 (2012).

[25] B. Juliá-Díaz, T. Zibold, M. K. Oberthaler, M. MeléMesseguer, J. Martorell, and A. Polls, Phys. Rev. A 86, 023615 (2012).

[26] In order to derive the second line of Eq. (12), we use the classical identity $\boldsymbol{\theta}^{(\mathrm{i} / \mathrm{f})}=\mp \partial R\left(\mathbf{n}^{(\mathrm{f})}, \mathbf{n}^{(\mathrm{i})}, t\right) / \partial \mathbf{n}^{(\mathrm{i} / \mathrm{f})}$ (minus for $\boldsymbol{\theta}^{(\mathrm{i})}$ and plus for $\left.\boldsymbol{\theta}^{(\mathrm{f})}\right)$ for each trajectory $\gamma$.

[27] In the truncated Wigner approach, the mean value of an operator $\hat{f}(t) \equiv f\left[\hat{a}_{\alpha}(t), \hat{a}_{\alpha}^{\dagger}(t)\right]$ is evaluated according to $\langle\hat{f}(t)\rangle=\int d^{L} \psi^{(0)} d^{L} \bar{\psi}^{(0)} P\left(\boldsymbol{\psi}^{(0)}, \overline{\boldsymbol{\psi}}^{(0)}\right) f\left[\psi_{\alpha}(t), \psi_{\alpha}^{*}(t)\right]$ where $\psi_{\alpha}(t)$ solves the classical equations of motion for the initial condition $\psi_{\alpha}(0)=\psi_{\alpha}^{(0)}=\left(\bar{\psi}_{\alpha}^{(0)}\right)^{*}$. In our case, $P\left(\boldsymbol{\psi}^{(0)}, \overline{\boldsymbol{\psi}}^{(0)}\right)$ corresponds to the manifold $\delta\left(\mathbf{n}^{(0)}-\mathbf{n}^{(\mathrm{i})}\right.$ ) (which is close to but not identical with the Wigner distribution of the initial Fock state) and $f$ corresponds to $\delta\left(\mathbf{n}-\mathbf{n}^{(\mathrm{f})}\right)$ according to Eq. (12), thus giving $\bar{P}^{\mathrm{cl}}$.

[28] Loop corrections [41] can be shown to vanish in this case.

[29] A. C. Cassidy, D. Mason, V. Dunjko, and M. Olshanii, Phys. Rev. Lett. 102, 025302 (2009).

[30] M. Lubasch, Master's thesis, Ruprecht-Karls-Universität Heidelberg, 2009.

[31] M. Hiller, T. Kottos, and T. Geisel, Phys. Rev. A 79, 023621 (2009).

[32] L. Tarruell, D. Greif, T. Uehlinger, G. Jotzu, and T. Esslinger, Nature (London) 483, 302 (2012).

[33] W. Bakr, J. Gillen, A. Peng, S. Fölling, and M. Greiner, Nature (London) 462, 74 (2009); J. Sherson, C. Weitenberg, M. Endres, M. Cheneau, I. Bloch, and S. Kuhr, Nature (London) 467, 68 (2010).

[34] J. E. Lye, L. Fallani, M. Modugno, D. S. Wiersma, C. Fort, and M. Inguscio, Phys. Rev. Lett. 95, 070401 (2005).

[35] Y.-J. Lin, R. Compton, K. Jimenez-García, J. Porto, and I. Spielman, Nature (London) 462, 628 (2009).

[36] In order to prevent three-body losses, it is preferable to avoid populations of more than two atoms per site.

[37] M. Gring, M. Kuhnert, T. Langen, T. Kitagawa, B. Rauer, M. Schreitl, I. Mazets, D. A. Smith, E. Demler, and J. Schmiedmayer, Science 337, 1318 (2012).

[38] T. Langen, R. Geiger, M. Kuhnert, B. Rauer, and J. Schmiedmayer, Nat. Phys. 9, 640 (2013).

[39] T. Geiger, T. Wellens, and A. Buchleitner, Phys. Rev. Lett. 109, 030601 (2012).

[40] S. Trotzky, Y.-A. Chen, A. Flesch, I. McCulloch, U. Schollwöck, J. Eisert, and I. Bloch, Nat. Phys. 8, 325 (2012).

[41] K. Richter and M. Sieber, Phys. Rev. Lett. 89, 206801 (2002); D. Waltner, Semiclassical Approach to Mesoscopic Systems (Springer, Berlin, 2012). 